\title{
DISTANCES AND ABSOLUTE MAGNITUDES OF A SAMPLE OF FAINT CATACLYSMIC VARIABLES
}

\author{
L. N. SPROATS ${ }^{1}$, S. B. HOWELL ${ }^{2}$, K. O. MASON ${ }^{1}$ \\ 1. Mullard Space Science Laboratory, Holmbury St. Mary, \\ Dorking, Surrey, RH5 6NT, England \\ 2. Astrophysics Group, Planetary Science Institute, \\ 620 N. 6th Ave, Tucson, AZ., USA
}

Our current knowledge of the basic characteristics of CVs, such as their distance and luminosity, are largely based on observations of optically bright $(V<16 \mathrm{mag})$ systems. This has tended to favour the more luminous members $\left(M_{\mathrm{V}} \sim 7 \ldots 10 \mathrm{mag}\right)$ lying nearby in the Galactic plane $(z<400 \mathrm{pc})$. In order to alleviate this bias, Howell \& Szkody (1990) compiled a list of $\sim 90 \mathrm{CVs}$ that were faint enough and were at high galactic latitude to potentially place them well outside the Galactic plane, in the halo. We have obtained infrared $J$ and $K$ band images of a number of the CVs listed by Howell \& Szkody (1990) in order to establish whether these systems are truely at large distances, or are instead a population of intrinsically faint objects lying relatively nearby. The distances were determined using Bailey's method (1982) and incorporated the recent $S_{K}$ calibrations by Ramseyer (1994).

We find that the majority of our sample are not at great distances but have $z<400 \mathrm{pc}$ with a distribution which is indistinguishable from their brighter well studied counterparts (from the compilation of Warner 1987; see Fig. 1). Only two stars in our sample (DO Leo and RU LMi) were found to have sufficiently large $z$ distances to constitute genuine halo candidates. The implication of this finding is that the CV space density in the Solar neighbourhood may be higher than previously anticipated.

While we find that the long period systems in our sample have $M_{\mathrm{V}}(\mathrm{min})$ consistent with the range derived by Warner (1987; see Fig. 2), the short period $(P<2 \mathrm{~h}) \mathrm{CVs}$ are up to 4 mag fainter in absolute magnitude when compared to those from Warner. The likely reason for their low luminosity is low mass transfer rates during minimum. However, the mean luminosity, averaged over their outburst cycle, is probably comparable to other systems of similar orbital period $\left(M_{\mathrm{V}}(\right.$ mean $\left.) \sim 7 \ldots 9 \mathrm{mag}\right)$. 


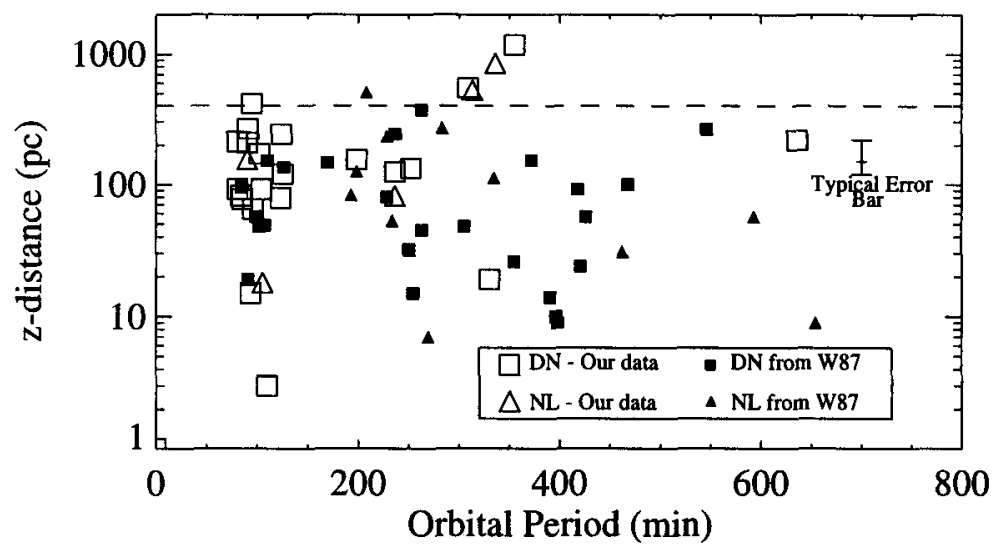

Figure 1. Distance above the Galactic plane for our sample of CVs plotted against orbital period. The mean $z$ distance of the old disk-halo population of stars as well as the bright CVs from Warner (1987) are also shown.

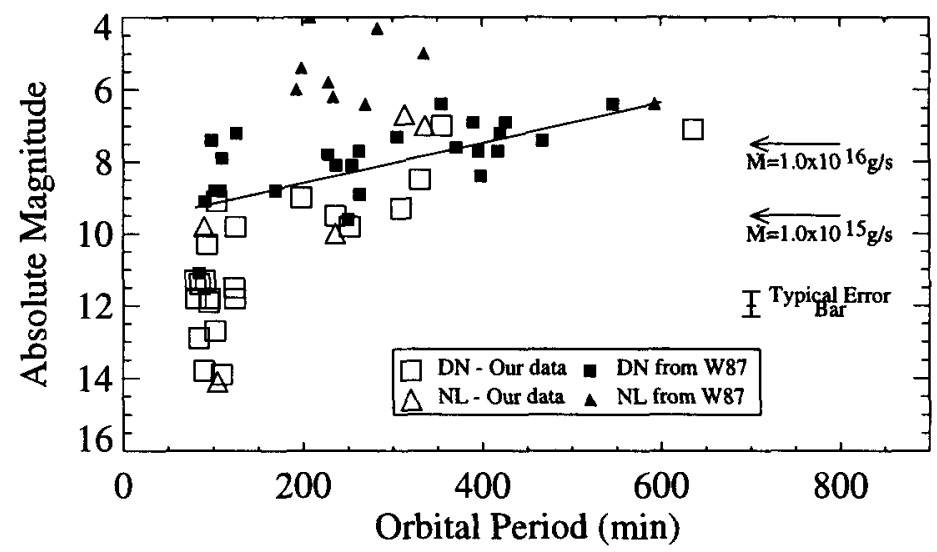

Figure 2. Absolute magnitude at minimum plotted against orbital period. Also plotted are approximate rates of mass transfer through the disk and the linear fit to dwarf novae from the Warner (1987) compilation.

\section{References}

Bailey, J., 1982, MNRAS, 197, 31

Howell, S.B., Szkody, P., 1990, Ap. J., 356, 623

Ramseyer, T.F., 1994, Ap. J., 425, 243

Warner, B., 1987, MNRAS, 245, 208 